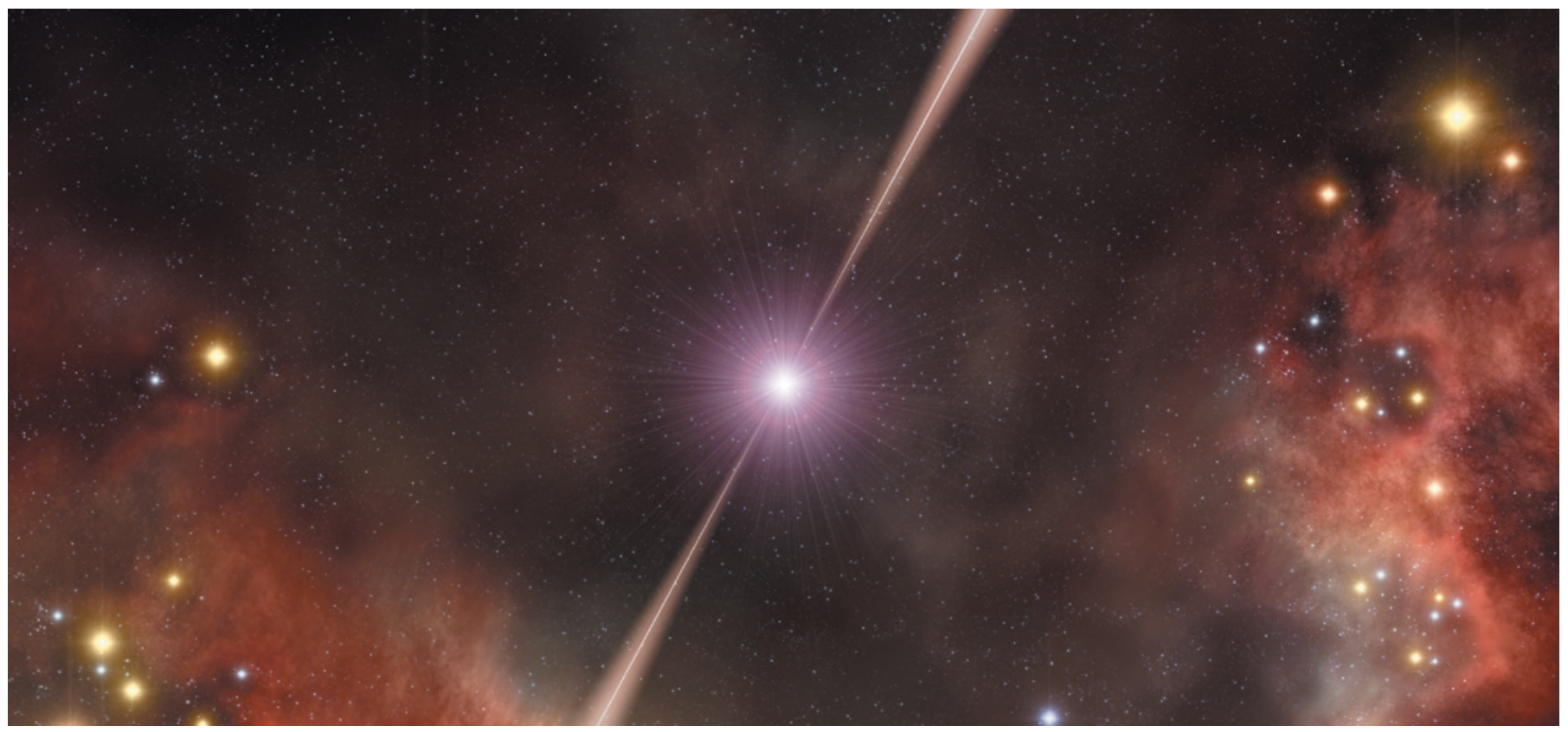

As their beams of intense light shine through surrounding gas, y-ray bursts like the one illustrated above pick up clues to the Universe's chemical evolution.

ASTROPHYSICS

\title{
Messages from the early Universe
}

\section{Bright and brief, $\gamma$-ray bursts hold clues to cosmic history.}

\section{BY ERIC HAND}

$\mathrm{U}$ nimaginably distant and powerful, the brief flashes of high-energy radiation known as $\gamma$-ray bursts (GRBs) were once one of astronomy's deepest mysteries. Now they are becoming a penetrating new tool. With orbiting observatories such as NASA's Fermi and Swift spacecraft routinely spotting the bursts, astronomers are laying plans to use them as cosmic flashbulbs to scrutinize the obscure details of the Universe's early years.

Seen almost daily, from all directions in space, GRBs are now thought to signal the collapse of a massive star's core into a black hole, an event that triggers a cataclysmic explosion. Their intense light can shine all the way across the visible Universe - bearing witness to the earliest chapters of its roughly 13-billion-year history. Theorists' understanding of the flashes is still evolving (see 'Flash of insight'), but at the 2012 Fermi/Swift GRB conference last week in Munich, Germany, astronomers discussed how they could use GRBs to chart the chemical evolution of the cosmos as light from the bursts is filtered through gas in the galaxies in which they reside.

Volker Bromm, an astronomer at the
University of Texas at Austin, says that GRBs are "cosmic Rosetta stones" that might even carry information about the composition of the Universe's very first stars, a few hundred million years after the Big Bang. "It almost has a metaphysical appeal," he says. "We want to go to the moment of first light."

Along with faint galaxies and quasars - the luminous cores of young galaxies with supermassive black holes at their centres - the objects that emit GRBs are among the most distant in the cosmos. As messengers from the early Universe, GRBs have advantages over the other two, says Nial Tanvir, an astronomer at the University of Leicester, UK. They are much brighter than distant galaxies, which means that a spectrograph has more information to work with when it splits a GRB's glow into its constituent wavelengths to reveal chemical absorption lines. And although quasars shine brightly, their light can be more erratic than that of GRBs and their spectra more complicated, which makes it more difficult to extract information about the material they have shone through.

The challenge is that GRBs are unpredictable and brief - typically
DNATURE.COM

For more from the early Universe, see: go.nature.com/azbtmo lasting only seconds at the highest energies. Their ephemeral flashes are followed by lingering afterglows that can be measured at longer wavelengths, but ground-based observatories must react quickly if they are to pick up the afterglows as soon as a spacecraft detects a burst. However, it can be done: one burst, detected by Swift in September 2005, was so bright that the 8-metre Subaru telescope on Hawaii detected the afterglow and obtained a spectrum more than three days later. With a measured redshift of 6.3 , the burst is estimated to have occurred when the Universe was less than $7 \%$ its current age. The spectrum, rich in detail, revealed that the re-ionization of hydrogen gas - a key turning point in cosmic history after the Universe cooled and darkened following the Big Bang - had been essentially complete.

But astronomers want to go even further back. GRBs have been going off ever since the formation of the Universe's first stars, which were probably massive, bright and short-lived. The light of such stars when they expire violently as GRBs would offer a coveted chemical fingerprint of the surrounding gas - the primordial stuff of the very early Universe.

By analysing GRBs in galaxies from different epochs, astronomers might be able to trace how the composition of the early Universe evolved, as early generations of stars burned a primordial supply of hydrogen and helium, converting it into heavier elements, collectively termed metals. "When did these big stars start making all these metals? When did they turn on?" asks Neil Gehrels, an astronomer at the Goddard Space Flight Center in Greenbelt, Maryland, and principal investigator for Swift.

To help to get a jump on early GRB observations, Jochen Greiner, an astronomer at the Max Planck Institute for Extraterrestrial Physics in Garching, Germany, and his team built 


\section{FLASH OF INSIGHT}

\section{What makes $\gamma$-ray bursts shine?}

Last week, astronomers at a conference in Munich, Germany, presented a new picture of the internal mechanics of $y$-ray bursts (GRBs), the fleeting but exceptionally luminous cones of light that jet outward along the rotation axes of stars as they collapse into black holes and explode as supernovae.

With only a flash to go on, the dissection of a GRB's fiery glow is a challenge to astronomers. For decades, theorists were convinced that most of a burst's $y$-rays originated with the shock waves that rush outward from the blast at nearly the speed of light. The twisted and compressed magnetic fields embedded within the shocks can accelerate electrons and cause them to emit $\mathrm{y}$-rays as synchrotron radiation.

But, as data accumulate, the evidence suggests that most of the $\mathrm{y}$-rays are emitted as thermal radiation at the scorchingly hot surface, or photosphere, of the exploding fireball (see 'Anatomy of a burst'). In such a scenario, GRBs shine in the same way as stars, through the collective motion of their constituent particles, but at energies that correspond to a temperature in the billions of degrees (see Nature http://doi.org/hwv; 2012).

"The rise of the photospheric model for me is transformative," says Julie McEnery, the project scientist for the Fermi y-ray space telescope at the Goddard Space Flight Center in Greenbelt, Maryland. "It's really a sea change."

Fermi, launched in 2008, has been instrumental in guiding this shift. It does not spot GRBs as precisely as the earlier satellite Swift, but it analyses the shape of a GRB spectrum across most of its $y$-ray energies. And the shape is not consistent with synchrotron radiation, says Sylvain Guiriec, a Fermi team member at Goddard. He says he has detected about a dozen spectra from bright GRBs that contain a small bump, a sign that thermal emissions are making a large contribution. E.H.

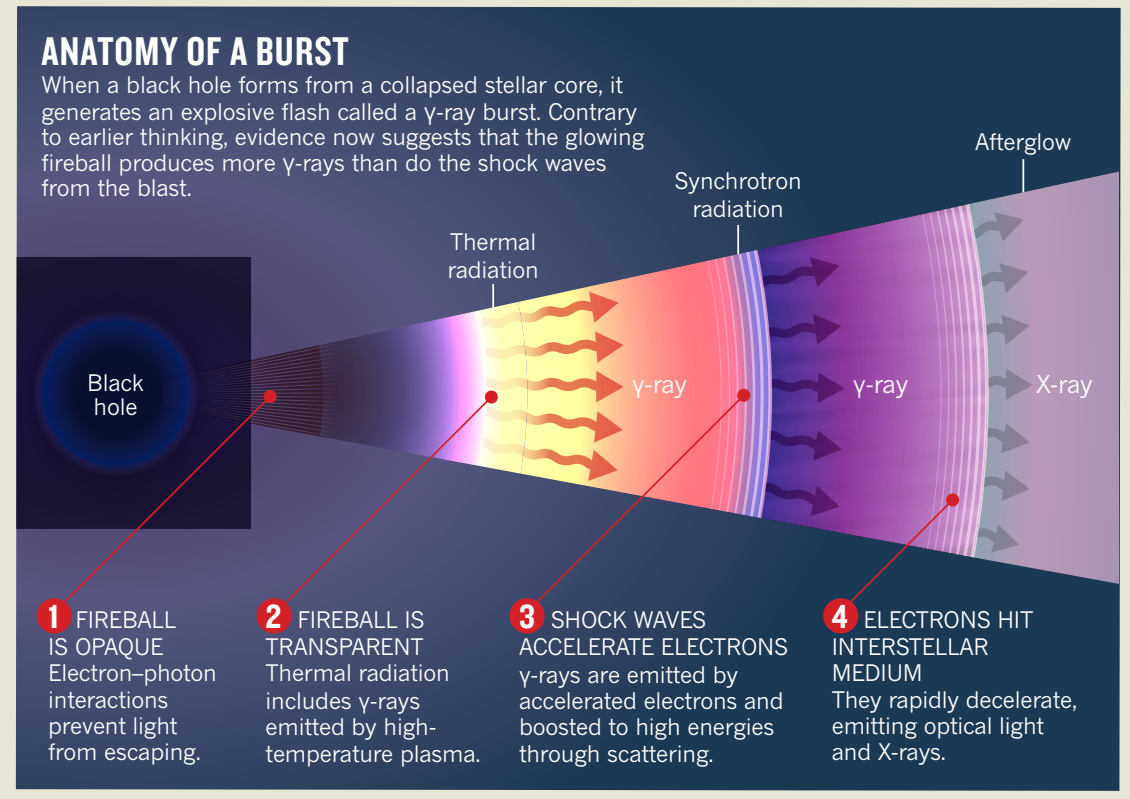

the Gamma-Ray Burst Optical/Near-infrared Detector (GROND) and added it to a 2.2-metre telescope operated by the European Southern Observatory (ESO) at La Silla in Chile. GROND responds to alerts from Swift, and seizes control of the ESO telescope. The automated system can make a quick estimate of a burst's distance; if the candidate is remote, Greiner and his colleagues call astronomers at the ESO's nearby Very Large Telescope, which has instruments that can make fine spectroscopic measurements. But Greiner is sometimes unable to convince them to interrupt their work. "They don't realize we have to react in minutes," he says.

Greiner also worries about the fact that Swift, although still working well, was designed to last only two years. Gehrels, however, is optimistic that with more spectrographs on ground-based telescopes, astronomers will be able to make the most of what Swift finds. He believes that it is just a matter of time before an explosion is detected that takes us even closer to the Big Bang. "All it's going to take is one burst," he says. "We just haven't got lucky yet." 